\title{
Monte Carlo Simulation of the Siemens Biograph Vision PET With Extended Axial Field of View Using Sparse Detector Module Rings Configuration
}

\author{
Sara A. Zein ${ }^{(}$, Nicolas A. Karakatsanis ${ }^{\circledR}$, Senior Member, IEEE, Maurizio Conti, \\ and Sadek A. Nehmeh, Member, IEEE
}

\begin{abstract}
We report on the NEMA-NU2-2012 performance of a hypothetical Monte Carlo (MC) model, Ex-PET, of the Siemens Biograph Vision positron emission tomography (PET)/CT (BioVis) with sparse detector module rings and extended axial field of view (AFOV). MC simulations were performed with the detector module rings interleaved with $32-\mathrm{mm}$ gaps, equivalent to the axial dimension of each detector module, yielding an AFOV of $48.0 \mathrm{~cm}$ (Bio-Vis has 25.6-cm AFOV). 3D-PET acquisition combined with a limited continuous-bed-motion (limited-CBM) was used to compensate for the loss in sensitivity within the gaps' regions. MC simulations of the Bio-Vis were performed for comparison purposes. All MC simulations were performed using GATE MC toolkit. Ex-PET exhibited 0.49, 0.16, and $0.16 \mathrm{~mm}$ deterioration in axial resolution at 1,10 , and $20 \mathrm{~cm}$ off-center of the transaxial field of view, respectively, compared to Bio-Vis. Only $1 \%$ reduction in system sensitivity and $6 \%$ reduction in peak NECR was observed with Ex-PET compared to Bio-Vis. 3D-OSEM image reconstruction, combined with $\mathrm{CBM}$, allowed compensating for the lack of counts within the gaps' regions. NEMA Image Quality test showed $<6 \%$ reduction in contrast recovery with Ex-PET versus Bio-Vis, yet the background variability was increased by up to $8 \%$. The feasibility of PET imaging with an easily adoptable sparse detector configuration was demonstrated. This can lay the pathway for future development of cost-effective PET systems with long and conventional AFOV's.
\end{abstract}

Index Terms-Continuous bed motion (CBM), extended axial field of view, Monte Carlo (MC), positron emission tomography (PET), sparse detectors.

Manuscript received July 27, 2020; revised October 7, 2020; accepted October 26, 2020. Date of publication October 29, 2020; date of current version May 3, 2021. (Sara A. Zein and Nicolas A. Karakatsanis contributed equally to this work.) (Corresponding author: Sara A. Zein.)

Sara A. Zein was with the Department of Radiology, Weill Cornell Medical College, New York, NY 10021 USA. She is now with Université de Bordeaux, CNRS/IN2P3, UMR5797, Centre d'Etudes Nucléaires de Bordeaux Gradignan, 33175 Gradignan, France (e-mail: zein@ cenbg.in2p3.fr).

Nicolas A. Karakatsanis is with the Department of Radiology, Weill Cornell Medical College, New York, NY 10021 USA (e-mail: nak2032@med.cornell.edu).

Maurizio Conti is with Siemens Medical Solutions Inc., Knoxville, TN 37932, USA (e-mail: maurizioconti@ siemens-healthineers.com).

Sadek A. Nehmeh is with the Department of Radiology, Weill Cornell Medical College, New York, NY 10021 USA (e-mail: san2028@med.cornell.edu).

Color versions of one or more figures in this article are available at https://doi.org/10.1109/TRPMS.2020.3034676.

Digital Object Identifier 10.1109/TRPMS.2020.3034676

\section{INTRODUCTION}

$\mathbf{P}$ OSITRON emission tomography (PET) is a molecular imaging technique that is widely used in neurology, cardiology, and oncology [1]. One major limitation of clinical PET systems is their relatively short axial field of view (AFOV), typically $<26 \mathrm{~cm}$ [2]-[7], hindering many applications that require simultaneous imaging of distant organs, such as imaging of the brain-heart axis [8], brain-gut axis [9], whole body kinetic parameters [10], etc. Recently, there has been an increased interest in extending the PET AFOV [11], [12]. The world's first total-body PET scanner, uEXPLORER (United Imaging, China), with $195 \mathrm{~cm}$ AFOV and $\sim 40$-fold higher sensitivity than the average sensitivity of current clinical PET systems with conventional AFOVs, has gained FDA approval [13]. Initial results from the uEXPLORER have shown the feasibility of PET imaging with ultralow dosage, ultrafast scans, total body compartmental kinetic modeling, etc. [13]. Total-body PET AFOVs have the potential to revolutionize the field of molecular imaging, enabling new important clinical and research PET applications. However, total-body PET systems are associated with significantly higher manufacturing costs mainly attributed to the high number of detector elements required to cover the long AFOV [14], thereby limiting the wide adoption of such innovative and useful technology.

In a more recent study, a 70-cm long PennPET Explorer was developed by the University of Pennsylvania comprising three ring-segments after expanding their original AFOV from 16.4 to $22.9 \mathrm{~cm}$ [15], [16]. Due to a firmware limitation, the readout was confined to the original $16.4-\mathrm{cm}$ AFOV per ring segment therefore resulting in two $7.4-\mathrm{cm}$ axial gaps between the three ring-segments and to a total active AFOV of $64 \mathrm{~cm}$. As the axial length of the two gaps was small compared to the total active AFOV, the gaps did not significantly affect volume sensitivity. The prototype demonstrated up to 9 times higher sensitivity than conventional PET systems with very comparable spatial resolution, good timing resolution of $256 \mathrm{ps}$ and excellent image quality (IQ). The same group recently studied the effect of axial gaps on the performance of long AFOV PET using simulations of multiple configurations of the Philips Vereos PET/CT system. 
Axial gaps of variable width $(4.3,7.6$, and $10.9 \mathrm{~cm})$ were introduced between multiple Vereos gantries of respective ring widths $(19.7,16.4$, and $13.2 \mathrm{~cm})$ by ignoring counts from the readout of various subsets of detector tile rings. In all configurations, the axial gaps width was smaller than that of the detector rings between the gaps. Spatial resolution and contrast recovery $(\mathrm{CR})$ were mildly impacted, and sensitivity and axial noise variation were reported especially with large gaps. Daube-Witherspoon et al. [17] concluded that axial gaps with widths less than one-half of the axial width of the detector rings between the gaps are preferable.

The feasibility of PET imaging with sparse detectors has been initially studied by Karakatsanis et al. where a sparse rings configuration was emulated by retrospectively removing real coincidence counts from list-mode clinical datasets acquired with the 64-ring Siemens Biograph mMR PET/MR (Erlangen, Germany). The counts were removed from all positions associated with any of the 32 even detector rings of the system. The resulting sparse data showed comparable IQ and lesion CR to those in the original clinical images [18]. However, the retrospective removal of counts from existing detector elements may not accurately reproduce the actual effects when physically removing the detectors. In another study, a long PET AFOV system (OpenPET) was introduced by incorporating a large axial gap of $\mathrm{W} \mathrm{cm}$ axial width between two ring-segments each of $\mathrm{W} \mathrm{cm}$ axial width [19]. One major drawback of the OpenPET configuration is the significant reduction in the transaxial field of view (FOV) after the introduction of the large axial gap in the center, due to the limited acceptance angle defined by the two PET ringsegments, and the decrease in system sensitivity in the large gap region between the two PET gantries [18], [20]. These effects were partially recovered in a subsequent study by the same group by introducing a smaller axial gap (shorter than the ring-segments axial width $\mathrm{W}$ ) within each of the two PET gantries [21], [22]. The same team developed a wholebody dual ring OpenPET with a $9 \mathrm{~cm}$ central gap for in beam charged particle therapy [23]. They also developed a single-ring OpenPET with a transformable gantry architecture adaptable for both open mode in-beam PET imaging where detector blocks are shifted axially and closed mode conventional PET imaging directly after irradiation to optimize performance [24].

An early study by Salomon et al. [25] investigated a sparse crystal setting in PET/MR systems to elongate the AFOV while exploiting the created axial gaps to introduce copper shielding against RF signals. Another study investigated an optimization-based reconstruction with image-total-variation constraint where the reconstructed data was collected by sparsely populated PET detectors configuration. The study mainly demonstrated the robustness and the potential usefulness of the optimization-based reconstruction for various data conditions in PET imaging especially in sparse detectors configurations [26]. A recent study by Zhang et al. reported on the feasibility of sparse PET imaging with either tangential or axial patterns of $50 \%$ detector reduction within each block. The sparsity of detectors was attained by excluding events from the listmode data of a precommercial Vereos, Philips release DPC PET/CTsystem (dPET) having a 1-to-1 LYSOto-SiPM coupling. Despite the $75 \%$ reduction in sensitivity and the drop in count rate, good IQ was maintained [27].

In this study, we propose to double the PET AFOV at no additional cost, by interleaving the PET detector module rings with uniform physical gaps of axial width equal to that of each detector module or block. This method, which is validated on the Siemens Biograph Vision geometry, is expected to yield nearly double the AFOV for the same number of detectors. The sparse ring configuration involves interleaving with uniform axial gaps between modules and thus is fundamentally different from our previous approach where the uniform axial gaps were much narrower and interleaved between detector elements of the same module or block [18], [28]. In this study, the gaps are smaller in number, but each has a significantly larger width. Our main motivation for proposing and evaluating the new sparse rings geometry is the ability to employ the same detector modules and associated readout electronics before and after the introduction of the axial gaps, thus facilitating the streamlined adoption of the new sparse module rings configuration to any commercial PET system with an original compact ring configuration. This design will not require significant hardware modification since the existing detector modules with their readout will not be individually modified but, instead, will only be spread out axially. The advantage of the sparse detector module configuration is that it can be retracted to a shorter AFOV for studies requiring high axial sensitivity making it more adaptable to specific imaging protocols.

A local gap (zero counts) in axial sensitivity per unit length of axial FOV (axial sensitivity profile) is expected at the interface between the gap and the physical detector regions when introducing gaps of width equal to that of a detector module and retain the limited number of detector module rings as that of modern commercial clinical PET systems. In order to eliminate the noise imbalance effects caused by these axial sensitivity gaps, we are proposing the novel application of the previously established continuous bed motion (CBM) PET scan technology [29], but along a limited axial distance approximately equal to only twice the gap axial width [limited continuous-bed-motion (limited-CBM)].

Moreover, the uniform distribution of equal and relatively small gaps between the detector modules is expected to balance the count sensitivity axially. The introduction of a larger number of gaps of uniformly smaller width would approximately yield the same AFOV length but would require modification of the detector elements configuration within the detector module. On the other hand, a lower number of gaps of a uniformly larger width is expected to enhance noise imbalance artifacts in the images between the gap and physical rings regions, due to the appearance of larger gaps in the scanner axial sensitivity profile. Furthermore, we chose this particular gap axial width to interleave between the detector module rings, as a smaller gap width would not provide sufficiently long AFOV while a larger gap width would diminish the IQ performance due to the more extensive gaps in the axial sensitivity profile and the associated more severe noise imbalance artifacts in the final images. 
We performed a Monte Carlo (MC) simulation of the Siemens Biograph Vision, called "Bio-Vis" in this work; the MC model of the Siemens Biograph Vision PET/CT (Bio-Vis) was extended, using this sparse detector module configuration, to a PET/CT scanner with double the axial length, called "ExPET" in this work. The sensitivity, spatial resolution, and IQ performance of proposed Ex-PET were validated using the NEMA NU-2 2012 guidelines, and compared against Bio-Vis.

\section{Methods And Materials}

\section{A. Monte Carlo Simulations}

MC simulations were carried out for two scanner geometries; Bio-Vis (gold standard) and Ex-PET. The GEANT4 application for tomographic emission (GATE) MC package [30], [31] was used to simulate the scanners' geometry, phantoms, sources, and physics processes. Bio-Vis was simulated using a cylindrical geometry of $41-$ and $43-\mathrm{cm}$ inner and outer radii, respectively, and it encompassed eight module rings, each with 38 detector modules. Each module consisted of 4 (transaxial) $\times 2$ (axial) mini-modules of $5 \times$ 5 Lutetium Oxyorthosilicate (LSO) detectors array. Each individual LSO crystal was $3.2 \mathrm{~mm} \times 3.2 \mathrm{~mm} \times 20 \mathrm{~mm}$. The crystal pitch was $3.2 \mathrm{~mm}$, thus resulting in detector modules size of $64 \mathrm{~mm}$ (transaxial) $\times 32 \mathrm{~mm}$ (axial) $\times 20 \mathrm{~mm}$ (depth) and a $25.6 \mathrm{~cm}$ long AFOV [Fig. 1(a)]. The Ex-PET model was simulated using the Bio-Vis geometry, but with the detector module rings interleaved with uniform physical gaps of $32 \mathrm{~mm}$ axial width, which is equal to the original axial width of each detector module [19], thus yielding a total of 15 detector module rings ( 8 physical interleaved with 7 virtual module rings) and a total physical axial scanner length of $48 \mathrm{~cm}$ [Fig. 1 (b)]. The axial gaps width was set no longer than the axial dimension of each detector module to avoid introducing too large gaps in the axial sensitivity profile that would impact IQ, as it was shown by Yamaya et al. [19]. The physics processes simulated in all cases are listed in Table I.

PET data for Bio-Vis were simulated using a $435-650 \mathrm{keV}$ energy window, a $4.7 \mathrm{~ns}$ coincidence time window (CTW), and a 320 ns paralyzable dead-time. The deadtime was optimized in order to reproduce the Siemens Biograph Vision system sensitivity that was reported by van Sluis et al. [6]. We considered an energy resolution of $11.7 \%$ in all the simulations of this study. The same specifications were applied for the Ex-PET configuration to allow the direct comparison of the two configurations performance under these common system settings.

\section{B. Continuous Bed Motion}

Bio-Vis data were acquired at a stationary bed position. However, all Ex-PET simulations were acquired in CBM mode, as per the Siemens FlowMotion option on Biograph PET scanners [29] but along a limited axial distance of $6.08 \mathrm{~cm}$, which is equal to the axial length of two detector modules minus a detector element, i.e., a distance of $20-1=19$ detector elements, (limited-CBM) to eliminate the gaps in the axial sensitivity profile, due to the physical gaps between the detector module rings, which may, in turn,

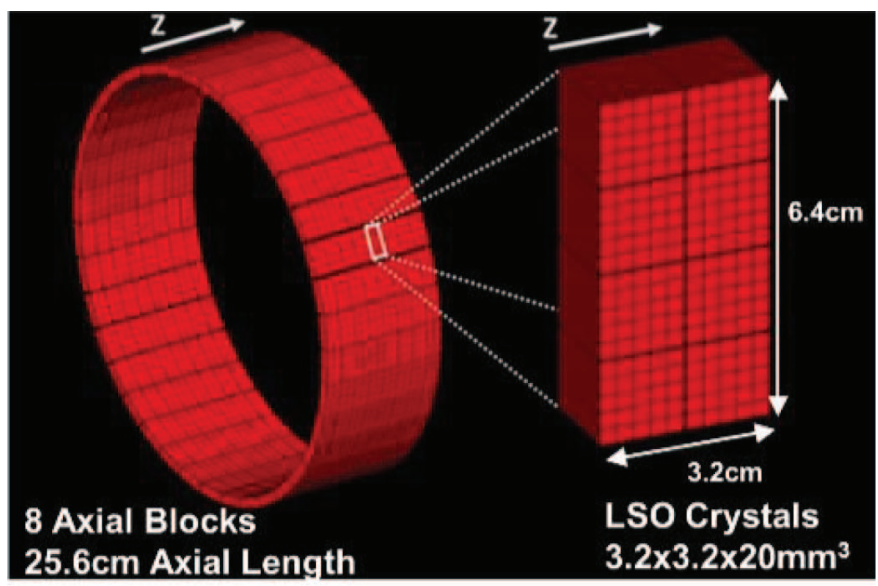

(a)

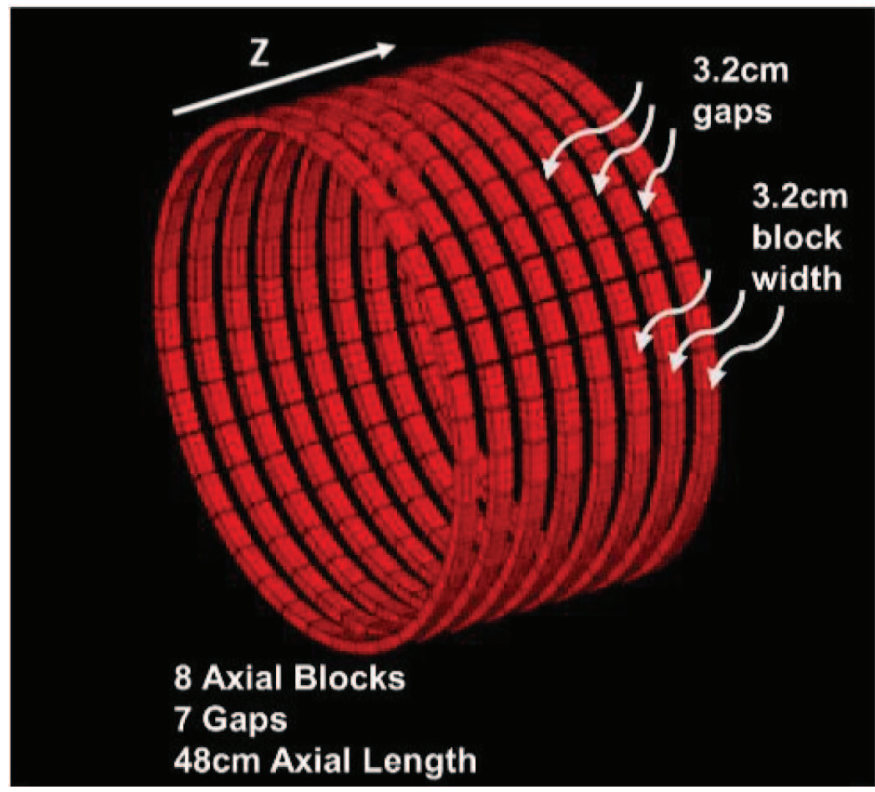

(b)

Fig. 1. Scanner geometries simulated in GATE. (a) Bio-Vis with eight compact LSO module rings. (b) Ex-PET with extended AFOV incorporating gaps of equal width as the detector modules.

TABLE I

InTERACTION Processes of Particles Simulated IN GATE BASEd on GEANT4 Physics Processes AND IMPLEMENTED MOdELS

\begin{tabular}{lll}
\hline \hline Particle & Process & Model \\
\hline Gamma & Photoelectric Effect & StandardModel \\
& Compton Scattering & StandardModel \\
& Rayleigh Scattering & PenelopeModel \\
Electron & Ionization & StandardModele- \\
& Bremsstrahlung & StandardModele- \\
& Multiple Scattering & MultipleScatteringe- \\
Positron & Ionization & StandardModele- \\
& Bremsstrahlung & StandardModele- \\
& Multiple Scattering & MultipleScatteringe- \\
& Positron Annihilation & \\
\hline \hline
\end{tabular}

cause severe noise imbalance and artifacts in the reconstructed images. For sparse detector module configuration, the 3-D PET acquisition in "limited-CBM" mode is a requirement even for 
single bed position studies. A CBM acquisition was emulated by following a step-and-shoot approach over 20 bed positions with a fixed scan time per step and a fixed axial step of $3.2 \mathrm{~mm}$ for each position, equal to a detector element crystal pitch. Thus, the bed was translated in a nearly continuous mode at a fixed speed by a total axial distance of $6.08 \mathrm{~cm}$, equal to the axial pitch of 19 detector elements, resulting in a total active AFOV of $54.1 \mathrm{~cm}$. At each step, listmode PET data were simulated for a fixed scan time $t_{\text {step }}=t_{\text {Bio- }}$ Vis $/ 20$, where $t_{\text {Bio- }}$ Vis is the respective Bio-Vis simulation time in stationary mode, i.e., without CBM. The listmode counts were then axially shifted to the first bed position and summed. The same step-wise acquisition approach was followed for the calculation of the CBM Ex-PET normalization factors.

\section{System Matrix}

The list-mode 3-D PET data were obtained using the ROOT object oriented data analysis framework [32] and binned into a 2-D matrix of sinogram planes, known as Michelogram. Each sinogram plane consisted of a 2-D array of 520 (radial) $\times 399$ (angular) detector pair bins. The Bio-Vis included 80 detector rings with the maximum ring difference (MRD) set to its maximum possible value of 79 according to the manufacturer. For the Ex-PET, the physical axial gaps in between the detector module rings were treated as virtual rings. The extension of the AFOV due to CBM by a distance equal to the crystal pitch of 19 detectors was accommodated by defining 19 additional "rings" in the Michelogram resulting in a total of 169 direct planes (80 physical, 70 virtual, and 19 additional rings). The MRD of Ex-PET was also set to its maximum possible value of 149. Time-of-flight (TOF) data were not simulated.

\section{Normalization Correction}

PET normalization data were simulated employing the same CBM scan mode. A uniform annular phantom, filled with a uniform activity concentration of $0.1 \mathrm{MBq}$ of ${ }^{18} \mathrm{~F}$, was used with $70 \mathrm{~cm}$ height, $79.9 \mathrm{~cm}$ inner diameter and $80 \mathrm{~cm}$ outer diameter. Normalization correction was performed using a component-based approach, as described by Casey et al. [33]. Specifically, four normalization components were calculated for each model: 1) geometric effect; 2) crystal interference; 3) crystal efficiency; and 4) axial effect. The inverse of the product of the four components was then calculated for each detector pair to compose the final normalization factors Michelogram for the Bio-Vis and Ex-PET systems. Normalization correction was finally applied within the 3-D ordered subsets expectation maximization (OSEM) reconstruction algorithm.

\section{E. System Performance}

The system spatial resolution, sensitivity, and IQ were evaluated for both the Bio-Vis (gold standard) and Ex-PET according to the NEMA NU-2 2012 guidelines [34].

\section{F. Spatial Resolution}

Bio-Vis Six ${ }^{18} \mathrm{~F}$ point sources of $1 \mathrm{~mm}$ diameter were simulated with water-equivalent density material and $9 \mathrm{MBq}$ activity. They were positioned at $(0.1 \mathrm{~cm}),(0.10 \mathrm{~cm})$, and $(0.20 \mathrm{~cm})$ locations at the AFOV center and in a transaxial slice at $1 / 8$ th AFOV distance from the AFOV edge for each scanner. Coincidence events were acquired for $60 \mathrm{~s}$ in both the Bio-Vis and the Ex-PET simulations. The listmode data were rebinned and reconstructed using the filtered back 3-D reprojection (FB3DRP) algorithm provided by the software for tomographic image reconstruction (STIR) [35], and with $1.65 \times 1.65 \times 1.6 \mathrm{~mm}^{3}$ voxel size. For each point source, the full width at half maximum (FWHM) and full width at tenth maximum (FWTM) of line profiles drawn through the hottest area within the corresponding activity distribution were measured in the $x, y$, and $z$ directions. The systems' tangential, radial, and axial spatial resolutions were finally calculated according to NEMA NU 2-2012 guidelines [34].

\section{G. Sensitivity}

Bio-Vis A $70 \mathrm{~cm}$ polyethylene tube of $1 \mathrm{~mm}$ diameter and $1 \mathrm{~mm}$ thickness was simulated with water equivalent density and $4 \mathrm{MBq}$ of ${ }^{18} \mathrm{~F}$. Five $70 \mathrm{~cm}$ long aluminum sleeves of $1.25 \mathrm{~mm}$ wall thickness and 6.4, 9.5, 12.7, 15.9, and $19.1 \mathrm{~mm}$ external diameters respectively, were added consecutively over the source tube and 100-s acquisitions were simulated for each added sleeve. The simulations were performed again at 10-cm off-center of the transaxial FOV for both models respectively. For each added sleeve, the total number of coincidences per slice is calculated using single-slice rebinning and then normalized by the source activity and total scan time [36]. The system sensitivity was defined as Sensitivity system $=$ $\sum_{i=0}^{n}$ sensitivity $_{\text {slice, } i}$, where sensitivity slice,$i_{i}$ is the sensitivity per slice $i$, and $\mathrm{n}$ is the total number of slices (159 for the Bio-Vis, and 339 for the Ex-PET). The system sensitivity versus the number of aluminum sleeves (attenuation degree) was fitted, and then extrapolated to zero attenuation to deduce the system sensitivity.

\section{H. Image Quality}

The IQ phantom was simulated using a cylindrical body shape $(14.7 \mathrm{~cm}$ radius and $21.4 \mathrm{~cm}$ height), thus modifying the shape of the background compartment of the original NEMA body IEC phantom due to a limitation in the definition of analytical geometrical shapes in GATE. No other modifications of the original NEMA IQ measurements protocol were introduced in the simulation. Similar to the NEMA IEC phantom, the simulated IQ phantom had six $1 \mathrm{~mm}$ thick plastic spheres with 10,13,17, 22, 28, and $37 \mathrm{~mm}$ internal diameters respectively, centered within the same plane, at $7 \mathrm{~cm}$ distance from the phantom edge. The background compartment was filled with water of $5.3 \mathrm{kBq} / \mathrm{ml}$ of ${ }^{18} \mathrm{~F}$, while the spheres were filled with water of $21.2 \mathrm{kBq} / \mathrm{ml}$ of ${ }^{18} \mathrm{~F}$, thus yielding to a target-to-background ratio (TBR) of 4:1. PET data acquisitions of a total of $10 \mathrm{~s}, 1 \mathrm{~min}, 3 \mathrm{~min}, 6 \mathrm{~min}$ and 9 min were simulated for the Bio-Vis and Ex-PET models.

PET images were reconstructed using the 3-D OSEM algorithm as implemented in STIR [35] with 19 subsets and normalization, randoms, scatter and attenuation correction. Randoms events were estimated using a delayed CTW. 
To evaluate IQ, the number of OSEM iterations was optimized for the Bio-Vis and Ex-PET independently to maximize the contrast-to-noise ratio (CNR) for the smallest sphere $(10 \mathrm{~mm})$ [37]

$$
\mathrm{CNR}=\frac{\bar{S}-\bar{B}}{\sigma_{B}}
$$

where $\bar{S}$ is the average number of counts in the region of interest (ROI) at the central slice of the sphere, and $\bar{B}$ and $\sigma_{B}$ are the mean and standard deviation of counts within a $60 \mathrm{~mm}$ diameter ROI in the background region of the phantom. The IQ and spheres CNRs were then evaluated for the two scanner models and for the different scan times using the corresponding optimum number of iterations. The percentage CR and background variability (BV) were also calculated for each sphere $i$ as follows:

$$
\begin{aligned}
\mathrm{CR}_{i} & =\frac{S_{i} / \overline{B k}_{i, 60}-1}{S_{i, \text { Act }} / B k_{i, \text { Act }}-1} \times 100 \\
\mathrm{BV}_{i} & =\frac{\sigma_{i, B k}}{\overline{B k}_{i, 60}} \times 100
\end{aligned}
$$

where $S_{i}$ is the mean number of counts inside a ROI drawn at the center of, and with the same diameter as, sphere $i$; $\overline{B k}_{60}$ is the average of the mean number of counts inside 60 background ROIs with diameter equal to that of sphere $i, 12$ of which were drawn on each of the transaxial slices at $0 \mathrm{~cm}, \pm 1 \mathrm{~cm}, \pm 2 \mathrm{~cm}$ distance from the spheres centers slice; $S_{i, \text { Act }} / B k_{i, \text { Act }}$ is the recovered TBR (ground truth $=4: 1$ ), and $\sigma_{i, B k}$ is the standard deviation of the mean counts of the 60 background ROIs.

\section{Noise Variability}

A $70-\mathrm{cm}$ long water cylinder with $14.7-\mathrm{cm}$ radius was simulated with a uniform $5.3 \mathrm{kBq} / \mathrm{ml}^{18} \mathrm{~F}$ activity concentration to study the noise characteristics of the Bio-Vis and Ex-PET reconstructed images. MC PET data were simulated for $9 \mathrm{~min}$ using the Bio-Vis and the Ex-PET models. The corresponding PET images were reconstructed with 3D-OSEM and normalization, randoms, scatters, and attenuation correction. A $10-\mathrm{cm}$ diameter ROI was drawn at the center of the cylinder in each of the 159 and 339 transaxial slices covering the phantom in the Bio-Vis and Ex-PET cases, respectively. The noise level in each transaxial slice was defined as

$$
N_{j}=\frac{\sigma_{j, B}}{\bar{B}_{j}}
$$

where $N_{j}$ is the noise level in slice $j$, and $\sigma_{j, B}$ and $\bar{B}_{j}$ are the standard deviation and mean counts within the ROI in slice $j$ respectively.

\section{J. Noise Equivalent Count Rate and Scatter Fraction}

The system count rate performance was evaluated as per NEMA NU-2 2012 guidelines. Specifically, a 70-cm long polyethylene cylinder with $20 \mathrm{~cm}$ diameter was used as a scatter phantom. A $70-\mathrm{cm}$ long line source at $45-\mathrm{mm}$ radial distance from the central axis of the polyethylene cylinder was simulated with $999 \mathrm{MBq}{ }^{18} \mathrm{~F}$ activity to study the noise equivalent count rate (NECR) and scatter fraction (SF) of the
Bio-Vis and Ex-PET configurations respectively. The phantom was positioned at the center of the scanner with its axis parallel to the axial direction in both configurations. Starting at high activity concentration, multiple consecutive 3-min PET data were acquired with 20 -min delays. Number of true $(T)$, scatter $(S)$, and random $(R)$ coincidences were registered for each time frame. SF and NECR were calculated according to NEMA NU-2 2012 [34].

\section{RESUlTS}

\section{A. Spatial Resolution}

Less than $2 \%$ difference in tangential and radial spatial resolutions were measured between the two scanner models (Table II). Axially, however, Ex-PET exhibited $\sim 19 \%$ degradation in spatial resolution at $1 \mathrm{~cm}$ off-center, compared to Bio-Vis. Some differences are noted from the measured quantities which may be attributed to the difference in the reconstruction algorithms. Measured data used filtered back projection after Fourier rebinning of sinograms [6] which is different from the FB3DRP used in this study.

\section{B. Sensitivity}

The system sensitivities for the Bio-Vis and Ex-PET as a function of aluminum thickness are displayed in Fig. 2. Less than $1 \%$ difference in system sensitivity was observed between the two scanner models (Table III). The corresponding axial sensitivity profiles at the center and $10 \mathrm{~cm}$ off-center are shown in Fig. 3. In stationary mode, Ex-PET exhibited a sharp reduction in sensitivity at the gaps between the detector module rings [Fig. 3(b)]. This was corrected by CBM [Fig. 3(c)].

\section{Image Quality}

CNR per OSEM iteration number of the smallest $10 \mathrm{~mm}$ sphere was calculated for Bio-Vis and Ex-PET, respectively, and in both cases the highest CNR is reached at the first iteration (each iteration represents a full cycle of 19 OSEM subiterations, one for each of the 19 view subsets) as Fig. 4 shows.

Fig. 5 shows transaxial and coronal images of the IQ phantom pertaining to the Ex-PET configuration with and without applying CBM. In stationary mode, the OSEM reconstruction is not sufficient to overcome the influence of the gaps resulting in visible image artifacts [Fig. 5(a) and (b)]. After applying CBM, the gap effect is fixed and all spheres, including the smallest $10-\mathrm{mm}$ sphere become clearly visible [Fig. 5(c) and (d)].

The transaxial images through the centers of the six spheres of the IQ phantom acquired with the Bio-Vis and Ex-PET CBM scans, respectively, for $10 \mathrm{~s}, 1,3,6$, and 9 min are displayed in Fig. 6(a). Fig. 6(b) shows the corresponding coronal slices through the center of the 10- and 28-mm diameter spheres. No image artifacts were observed in all cases. IQ improved with increasing scan time, and all six spheres, including the smallest, were detectable with both scanner configurations with 6-min scan time. The IQ images corresponding to the first iteration showed the highest CNR for the 10-mm diameter sphere in both Bio-Vis and Ex-PET, and therefore all subsequent images 
TABLE II

SPATIAL RESOlution

\begin{tabular}{|c|c|c|c|c|c|c|}
\hline \multirow[b]{2}{*}{ Tangential } & \multicolumn{2}{|l|}{ Bio-Vis } & \multicolumn{2}{|l|}{ Ex-PET } & \multicolumn{2}{|c|}{ Siemens Biograph Vision ${ }^{\mathrm{TM}}$} \\
\hline & FWHM (mm) & FWTM $(\mathrm{mm})$ & FWHM (mm) & FWTM (mm) & FWHM (mm) & FWTM (mm) \\
\hline $1 \mathrm{~cm}$ & $3.75 \pm 0.01$ & $7.57 \pm 0.02$ & $3.74 \pm 0.00$ & $7.57 \pm 0.01$ & 3.7 & 7.2 \\
\hline $10 \mathrm{~cm}$ & $5.19 \pm 0.02$ & $11.82 \pm 0.06$ & $5.11 \pm 0.01$ & $11.91 \pm 0.08$ & 3.9 & 7.3 \\
\hline $20 \mathrm{~cm}$ & $6.19 \pm 0.04$ & $18.34 \pm 0.11$ & $6.07 \pm 0.08$ & $17.61 \pm 0.27$ & 3.6 & 7.0 \\
\hline \multicolumn{7}{|l|}{ Radial } \\
\hline $1 \mathrm{~cm}$ & $3.91 \pm 0.01$ & $8.75 \pm 0.03$ & $3.98 \pm 0.01$ & $8.88 \pm 0.04$ & 3.7 & 7.4 \\
\hline $10 \mathrm{~cm}$ & $4.62 \pm 0.01$ & $8.62 \pm 0.01$ & $4.66 \pm 0.02$ & $8.71 \pm 0.04$ & 4.6 & 8.8 \\
\hline $20 \mathrm{~cm}$ & $5.23 \pm 0.03$ & $9.10 \pm 0.06$ & $5.27 \pm 0.04$ & $9.16 \pm 0.03$ & 6.0 & 11.1 \\
\hline \multicolumn{7}{|l|}{ Axial } \\
\hline $1 \mathrm{~cm}$ & $2.56 \pm 0.01$ & $5.9 \pm 0.04$ & $3.05 \pm 0.02$ & $6.16 \pm 0.03$ & 3.8 & 7.6 \\
\hline $10 \mathrm{~cm}$ & $3.51 \pm 0.01$ & $7.2 \pm 0.01$ & $3.67 \pm 0.01$ & $7.19 \pm 0.03$ & 4.3 & 9.2 \\
\hline $20 \mathrm{~cm}$ & $5.51 \pm 0.06$ & $9.61 \pm 0.05$ & $5.67 \pm 0.06$ & $10.15 \pm 0.15$ & 4.6 & 10.2 \\
\hline
\end{tabular}

Spatial resolution FWHM and FWTM in the Bio-Vis and Ex-PET configurations and the published spatial resolution of Siemens Biograph Vision ${ }^{\mathrm{TM}}$ as reported by van Sluis et al. [6]

Standard error is calculated as standard deviation of three measurements from three different 1 min simulations.

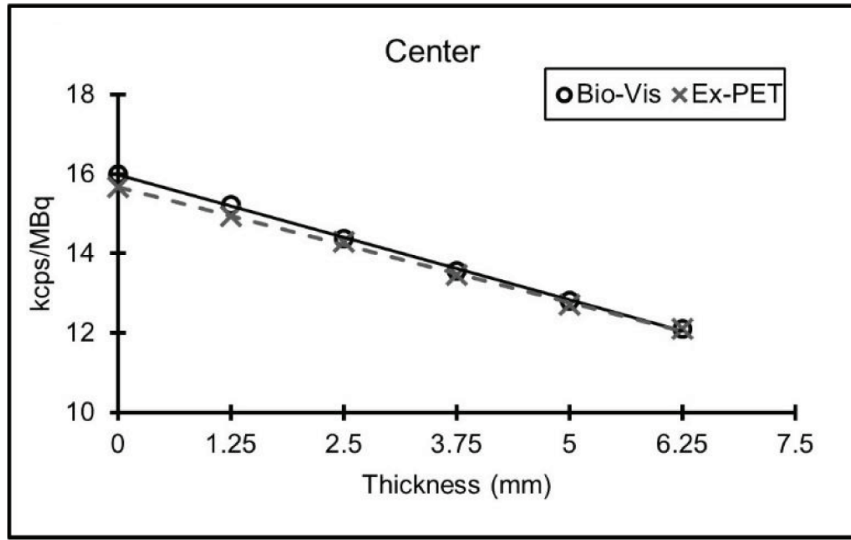

(a)

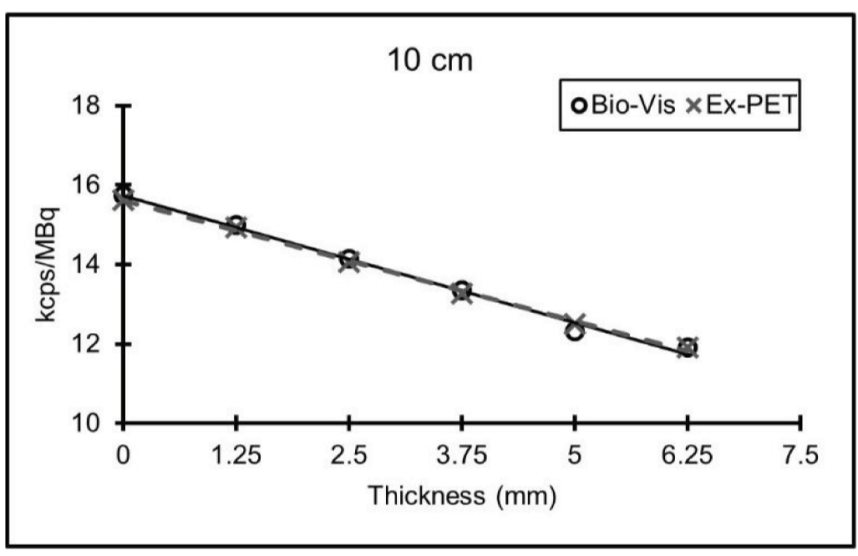

(b)

Fig. 2. System sensitivity per aluminum thickness interpolated to zero attenuation for Bio-Vis and Ex-PET at the center (a) and $10 \mathrm{~cm}$ off-center (b) of the transaxial FOV.

were reconstructed using one iteration. Fig. 7 shows the CNR as a function of scan time for each of the spheres and for both the Bio-Vis and Ex-PET configurations. Ex-PET exhibited consistently lower CNR compared to Bio-Vis for all spheres and at all acquisition times. Quantitative analysis was performed using the 9-min acquisition images. Ex-PET showed less than $6 \%$ difference in percent CR (Table IV), and up to $8 \%$ increase in the BV for the 22-mm diameter sphere ROI's (3.18 for BioVis versus 3.44 for Ex-PET) (Table V) compared to Bio-Vis.

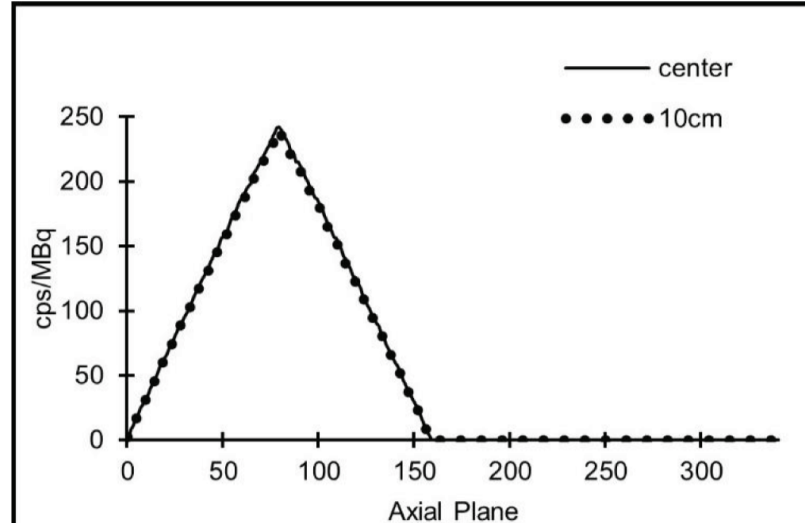

(a)

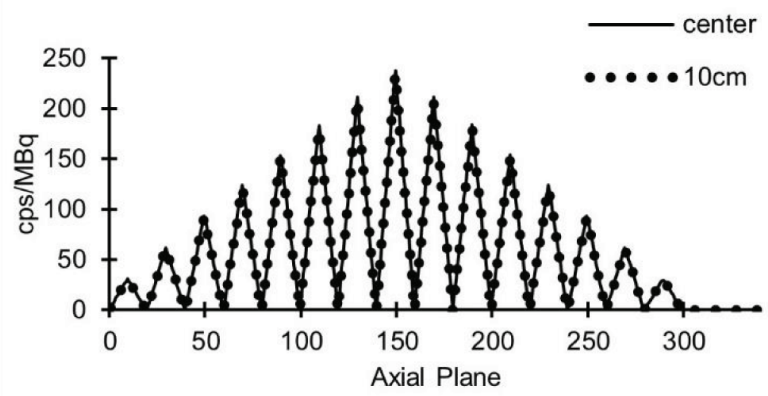

(b)

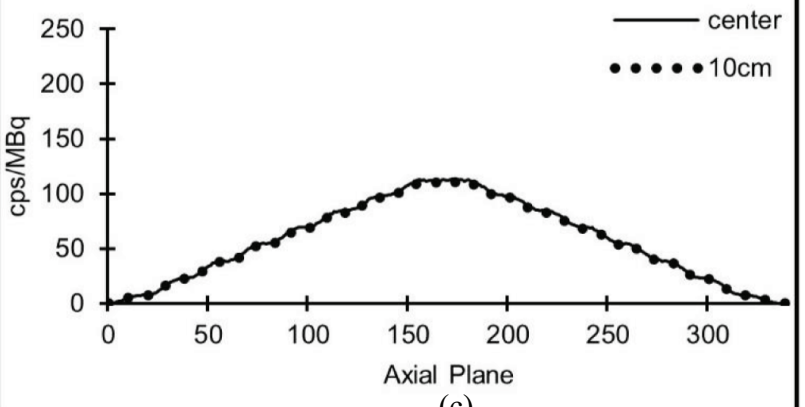

(c)

Fig. 3. Sensitivity profiles at the center and $10 \mathrm{~cm}$ off-center for (a) Bio-Vis, (b) Ex-PET with 1 stationary bed position, and (c) Ex-PET with CBM.

For each of the six sphere ROIs, the BV difference between the two scanner models was statistically insignificant (two-tailed paired t-test showed $p>0.05$ ). 
TABLE III

SENSITIVITY

\begin{tabular}{cccc}
\hline \hline & Bio-Vis & Ex-PET & $\begin{array}{c}\text { Siemens } \\
\text { Biograph Vision }^{\mathrm{TM}}\end{array}$ \\
\hline Center & $15.96 \pm 0.01$ & $15.68 \pm 0.01$ & 16.4 \\
$10 \mathrm{~cm}$ & $15.74 \pm 0.01$ & $15.61 \pm 0.04$ & 16.3 \\
\hline \hline
\end{tabular}

System sensitivity (kcps/MBq) in the Bio-Vis and Ex-PET configurations and the published sensitivity of Siemens Biograph Vision ${ }^{\mathrm{TM}}$ as reported by van Sluis et al. [6]

Standard error is calculated as standard deviation of three measurements from three different $100 \mathrm{~s}$ simulations.

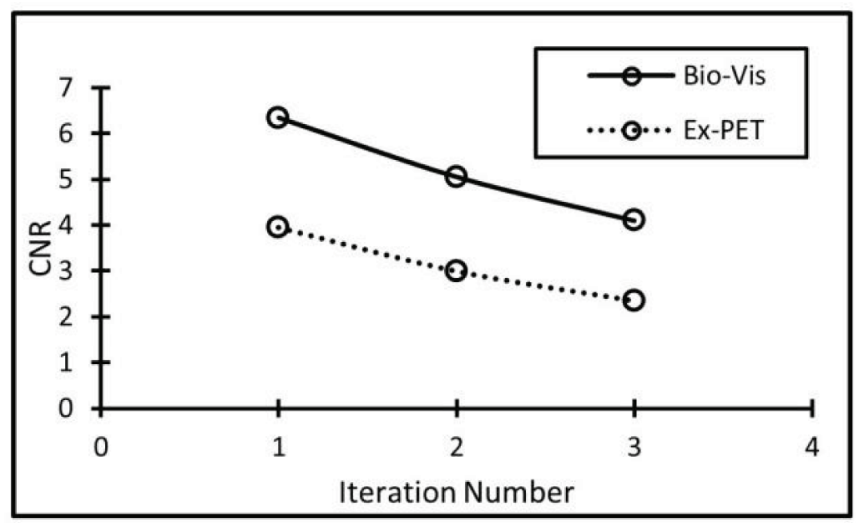

Fig. 4. CNR of the $10 \mathrm{~mm}$ sphere as a function of the number of full cycles of OSEM iterations $(1$ iteration $=1$ full cycle of 19 subiterations, 1 subiteration per view subset) for the Bio-Vis and Ex-PET configurations, using the image set corresponding to the 9-min simulation time where the first full cycle of iterations exhibited the maximum CNR for both configurations.

\section{Noise Equivalent Count Rate and Scatter Fraction}

Fig. 8 shows the NECR for Bio-Vis (black diamonds) and Ex-PET (red diamonds). Ex-PET exhibited a reduced NECR of up to $6 \%$ compared to Bio-Vis. Table VI shows the SF and NECR peaks for Bio-Vis and Ex-PET as well as measured values on Siemens Biograph Vision as reported by van Sluis et al. [6]. The two scanner configurations exhibited a similar SF of $34 \%$.

\section{DISCUSSION}

Long AFOV PET imaging has been gaining significant interest recently because of its wide scope of potential clinical applications [11], such as multiorgan simultaneous imaging (e.g., heart-brain axis [8], gut-brain axis [9], etc.), simultaneous multiorgan kinetic modeling [10], breath-hold imaging [38], new drug dosimetry studies, etc. Long PET AFOVs with compact ring configurations yield a multifold increase in system sensitivity due to the respective increase in the number of detectors [13], [39]. A major limitation for the wide adoption of such technology is, however, the multifold increase in the manufacturing costs due to the large number of detectors required to cover the long AFOV in a compact detector configuration.

In this study, we have demonstrated a cost-effective and commercially translatable solution to extend the PET AFOV by more than $100 \%$ using a sparse detector module rings geometry and bed motion. In the proposed geometry, the

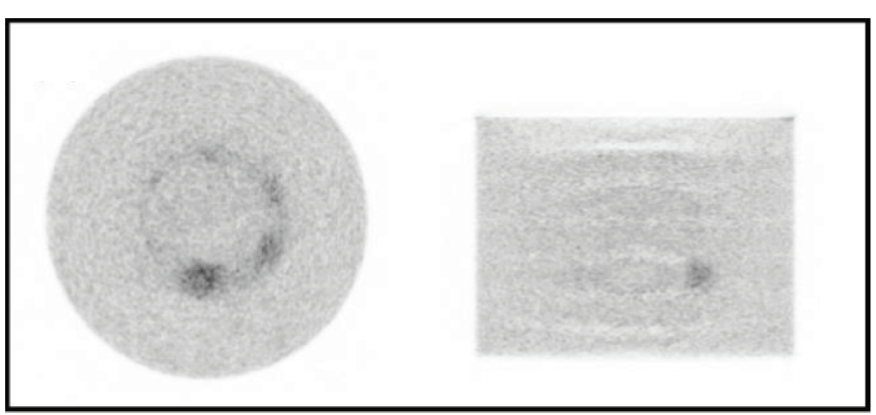

(a)

(b)

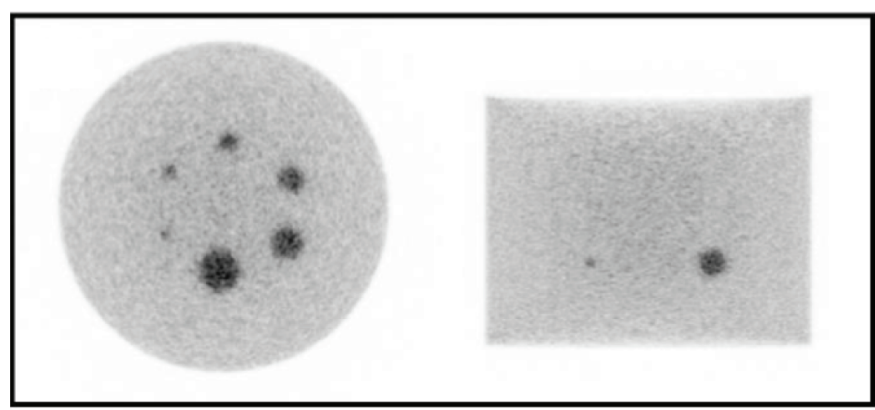

(c)

(d)

Fig. 5. IQ phantom OSEM image reconstruction with 1 iteration and 19 subsets of $431 \times 431$ image matrix $1.65 \mathrm{~mm} \times 1.65 \mathrm{~mm}$ pixel and $1.6-\mathrm{mm}$ slice thickness for a total 9-min duration applying normalization and attenuation correction as implemted in STIR [35]. Image artifacts are visible in both transaxial (a) and coronal (b) views with Ex-PET and stationary one bed position of the IQ phantom. When applying CBM with Ex-PET, artifacts no longer exist in both transaxial (c) and coronal (c) views and all spheres, including the smallest $10 \mathrm{~mm}$ sphere are clearly visible.

detectors' architecture within both each module and each ring were kept intact between the compact and sparse configurations, facilitating the streamlined adoption of the proposed sparse geometry to any of the current generation clinical PET systems. Moreover, as the manufacturing cost is mostly determined by the number of scintillation and optical detector modules and readout electronic boards, the proposed configuration is not expected to raise the manufacturing cost significantly. This is a major improvement relative to our past approach where the smaller gaps introduced between individual scintillating detector elements within each block or module would retain the original number of scintillation detector modules but also would yield a significant increase in the number of optical detector modules (e.g., SiPMs or APDs) required [18]. On the other hand, it should be noted that for certain PET systems detector modules belonging to different rings may be interconnected or integrated within a larger structure (e.g., ring sectors) thus preventing the axial separation of these detector modules and the introduction of axial gaps between them.

Regarding the choice of the CTW width, we opted to use a 4.7-ns CTW for both systems, which is our estimated value for the Bio-Vis geometry, despite the significantly larger AFOV of the Ex-PET. The maximum CTW for Ex-PET would theoretically need to be $8.6 \%$ wider compared to that of BioVis, to accommodate the longer line-of-response (LOR) length corresponding to the Ex-PET larger maximum acceptance 

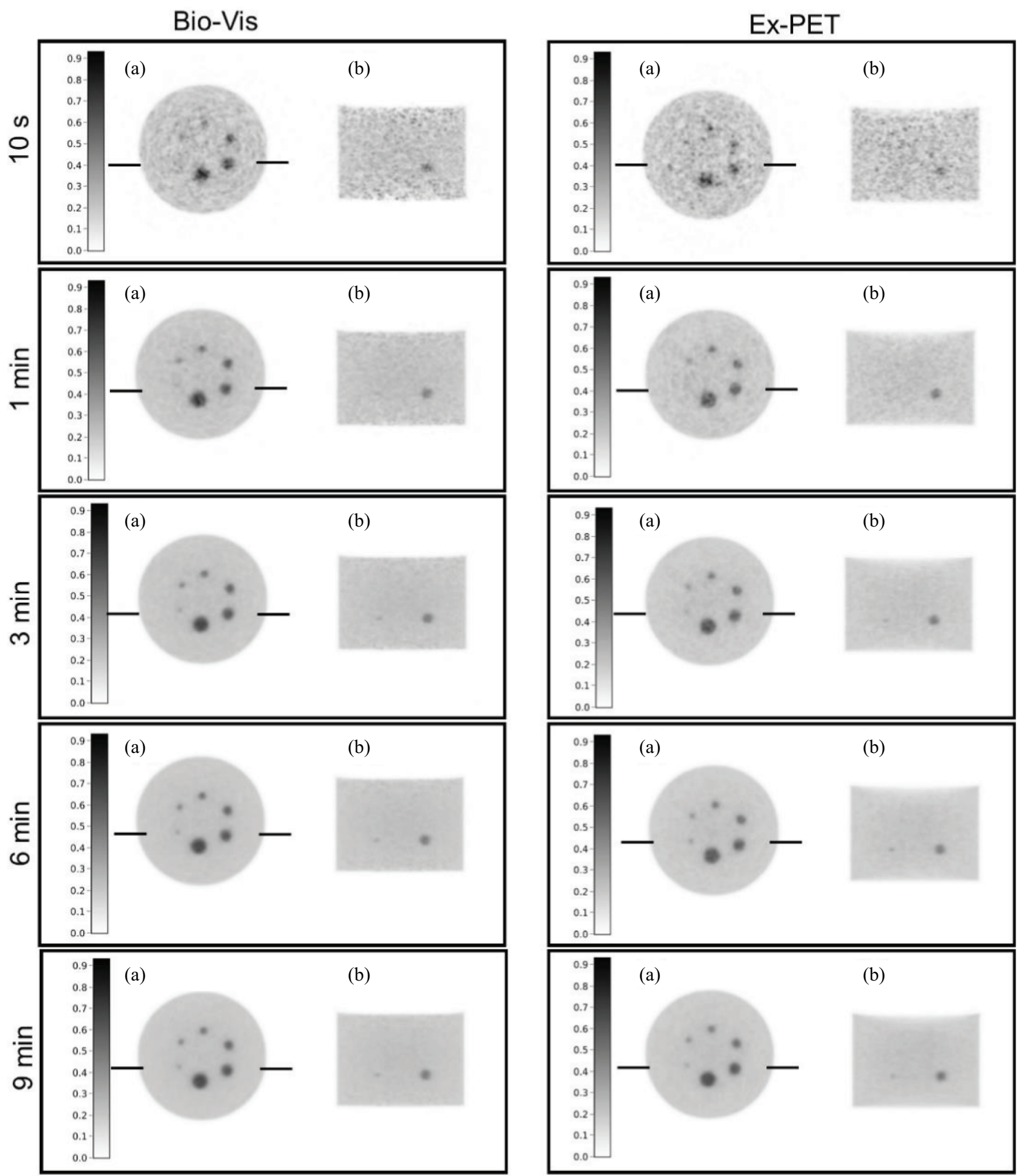

Fig. 6. Transaxial (a) and coronal (b) views of the simulated IQ phantom reconstructed images using the Bio-Vis configuration (left) and the Ex-PET configuration (right) for $10 \mathrm{~s}, 1 \mathrm{~min}, 3 \mathrm{~min}, 6 \mathrm{~min}$, and $9 \mathrm{~min}$ simulation scan time. The bars in figures (a) show the cross section corresponding to the coronal view. (3-D OSEM reconstruction with normalization and attenuation correction as implemented in STIR [35], one iteration, 19 subsets, $431 \times 431$ image matrix with $1.65 \mathrm{~mm} \times 1.65 \mathrm{~mm}$ pixel size, $1.6 \mathrm{~mm}$ slice thickness, $3.2 \mathrm{~mm} \times 3.2 \mathrm{~mm} \times 3 \mathrm{~mm}$ FWHM Gaussian post-filter, 159 and 339 transaxial slices for the Bio-Vis with one bed position and the Ex-PET with CBM, respectively).

angle of $30.4^{\circ}\left(17.6^{\circ}\right.$ in the case of Bio-Vis) [40]. Such a long CTW would avoid the rejection of coincidence data originating from locations at the edges of both the transaxial and axial FOV when detected for this particularly large acceptance angle, as suggested by Poon et al. [40]. Nevertheless, the data detected at such large axial acceptance angles are 


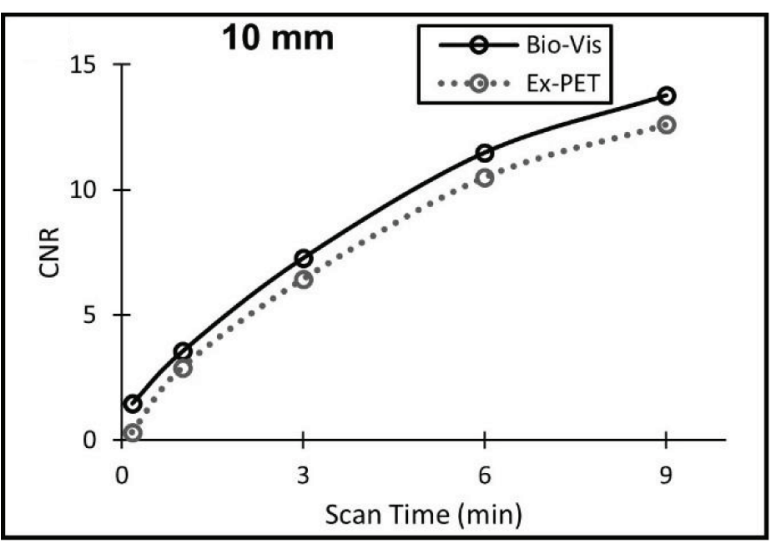

(a)

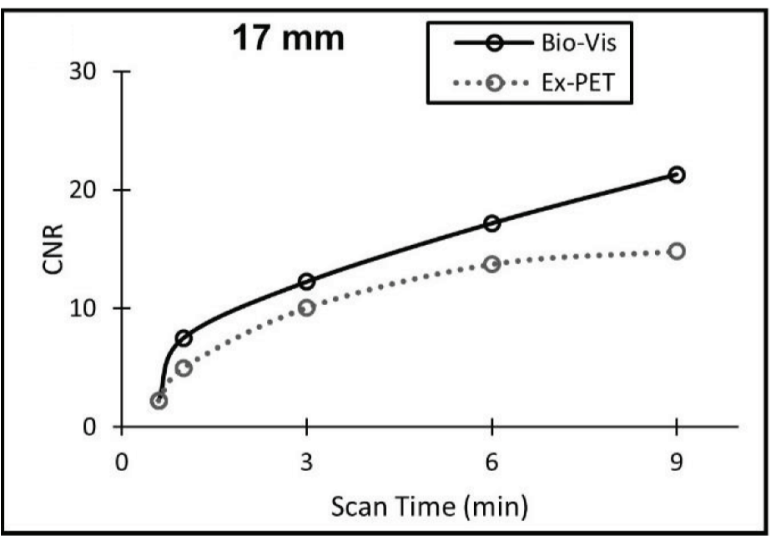

(c)

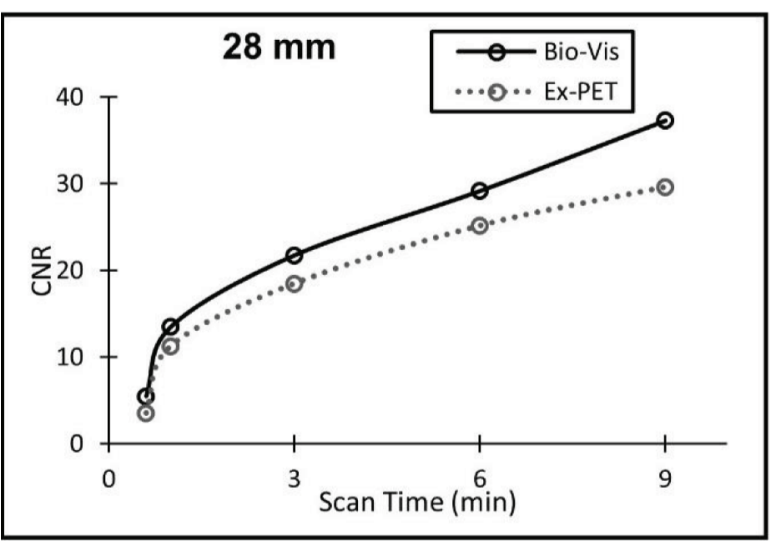

(e)

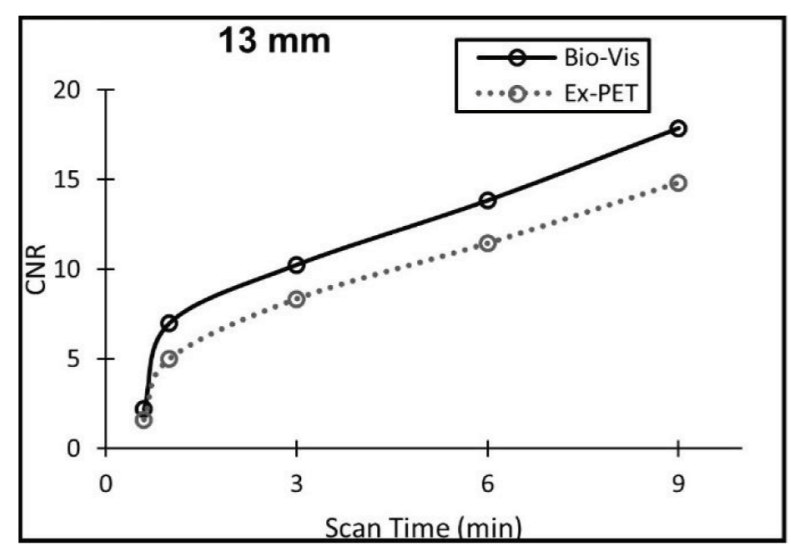

(b)

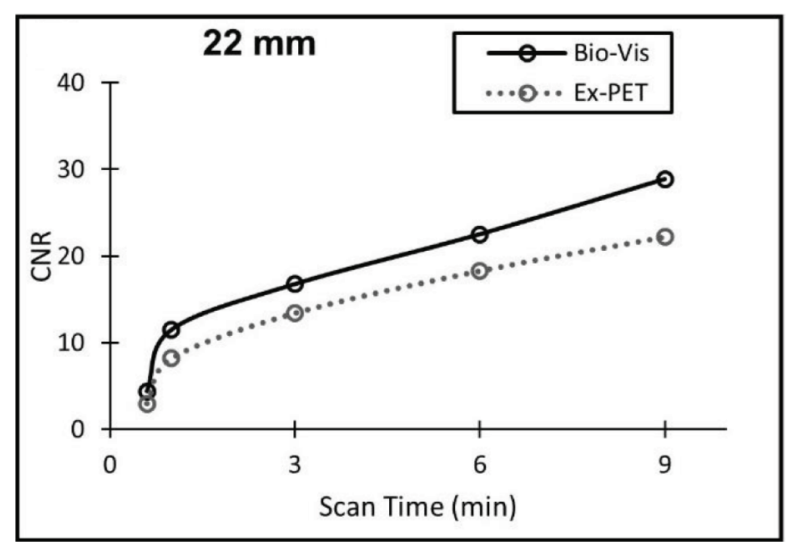

(d)

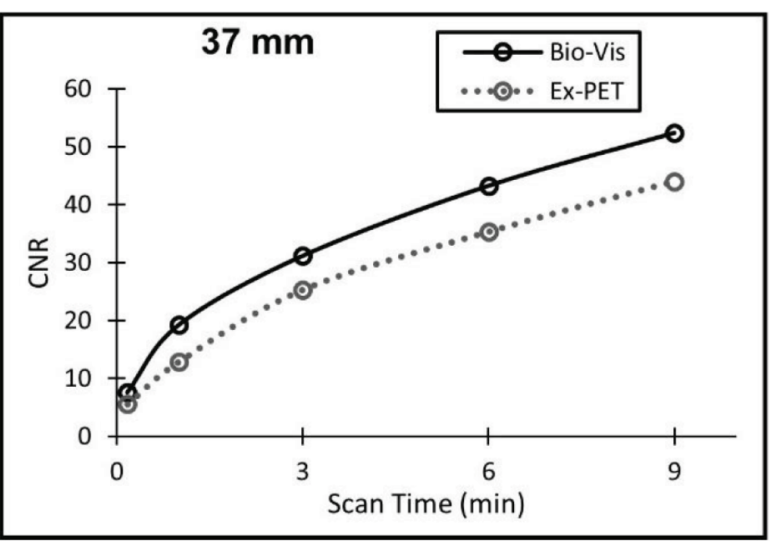

(f)

Fig. 7. CNR in Bio-Vis and Ex-PET configurations: CNR per scan time for (a) $10 \mathrm{~mm}$, (b) $13 \mathrm{~mm}$, (c) $17 \mathrm{~mm}$, (d) $22 \mathrm{~mm}$, (e) $28 \mathrm{~mm}$, and (f) $37 \mathrm{~mm}$ diameter spheres after one iteration.

TABLE IV

PERCENTAGE CR

\begin{tabular}{llllll}
\hline \hline Diameter & $10 \mathrm{~mm}$ & $13 \mathrm{~mm}$ & $17 \mathrm{~mm}$ & $22 \mathrm{~mm}$ & $28 \mathrm{~mm}$ \\
\hline Bio-Vis (\%) & $24.80 \pm 1.93$ & $36.26 \pm 2.33$ & $44.22 \pm 2.54$ & $50.91 \pm 1.74$ & $59.32 \pm 0.92$ \\
Ex-PET (\%) & $23.89 \pm 4.03$ & $34.08 \pm 2.30$ & $44.47 \pm 0.27$ & $52.23 \pm 1.34$ & $61.64 \pm 2.09$ \\
\hline \hline
\end{tabular}

Percentage contrast recovery of the six hot spheres regions of interest in the Bio-Vis and Ex-PET cases

Standard error is calculated as standard deviation of three measurements from three different 9 min simulations.

usually associated with relatively low sensitivity due to the large angle of incidence of the photon paths to the detectors along the planes corresponding to these axial acceptance angles. Thus, the use of narrower CTW would yield a relatively small percentage of rejected coincidence events that is not expected to contribute significantly to the already high 
TABLE V

PERCENTAGE B V

\begin{tabular}{llllll}
\hline \hline Diameter & $10 \mathrm{~mm}$ & $13 \mathrm{~mm}$ & $17 \mathrm{~mm}$ & $22 \mathrm{~mm}$ & $28 \mathrm{~mm}$ \\
\hline Bio-Vis (\%) & $5.38 \pm 0.27$ & $4.48 \pm 0.67$ & $3.99 \pm 0.76$ & $3.18 \pm 0.07$ & $2.79 \pm 0.12$ \\
Ex-PET (\%) & $4.99 \pm 2.12$ & $4.31 \pm 0.86$ & $3.76 \pm 0.77$ & $3.44 \pm 0.58$ & $2.48 \pm 0.18$ \\
\hline \hline
\end{tabular}

Percentage background variability of the six hot spheres regions of interest in the Bio-Vis and Ex-PET cases

Standard error is calculated as standard deviation of three measurements from three different 9 min simulations.

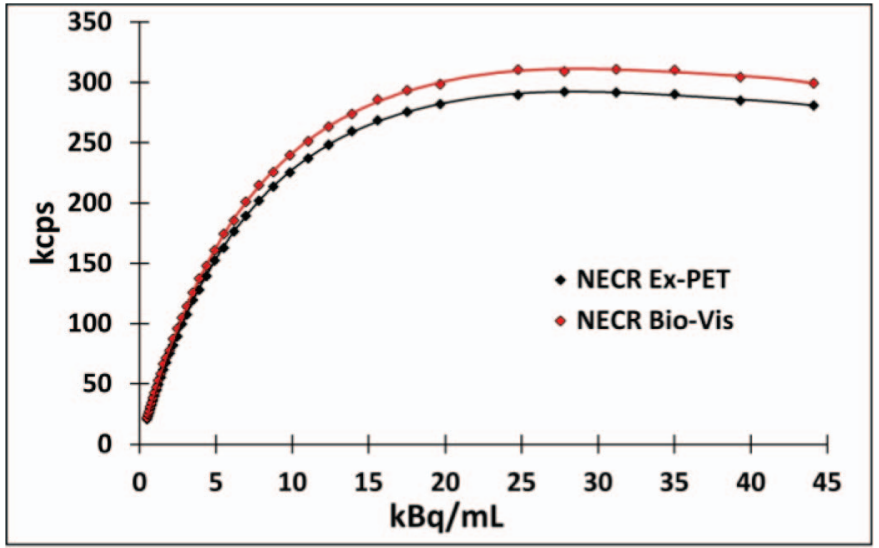

Fig. 8. NECR plots of Bio-Vis and Ex-PET.

levels of noise expected at these locations of the FOV. In the meantime, the proper 3-D normalization of the PET acquisition would eliminate any artifacts on the IQ at these locations as a result of the elimination of these events. On the other hand, such a CTW would be larger than necessary for all the rest of the LOR data detected at smaller axial acceptance angles with a higher sensitivity thereby yielding a significantly higher randoms fraction compared to that expected with the narrower CTW of Bio-Vis. An optimal solution may therefore be the application of an adaptive CTW determined by the acceptance angle of each LOR's azimuthal plane. However, we have elected to apply the Bio-Vis CTW for the Ex-PET system too, in order to avoid the overall elevation of the randoms coincidence fraction, retain a simple approach of a system-wide CTW and allow the direct comparison between the two scanner configurations under a common CTW.

Although the axial parallax effect can yield a deterioration in the axial spatial resolution, it is expected to have limited effect on the IQ when using iterative 3-D image reconstruction methods, as it was shown by Schmall et al. [41]. In Ex-PET, doubling the AFOV increased the axial acceptance angle from $\pm 17.6^{\circ}$ (Bio-Vis) to $\pm 30.4^{\circ}$ (Ex-PET). This apparently has yielded a $19 \%$ deterioration in Ex-PET axial spatial resolution at the center of the transaxial FOV, improved to less than 5\% and 3\% difference, compared to those of the Bio-Vis at $10 \mathrm{~cm}$ and $20 \mathrm{~cm}$ off-center, respectively. The longer AFOV had, however, no significant impact $(<2 \%$ deterioration) in Ex-PET transaxial spatial resolution (Table II). Similar results were shown by Schmall et al. by increasing the acceptance angle from $\pm 12^{\circ}$ to $\pm 67^{\circ}$ [41]. The larger deterioration in axial resolution in our study, compared to what was reported by Schmall et al. for similar axial acceptance angles, can be the result of increased axial parallax

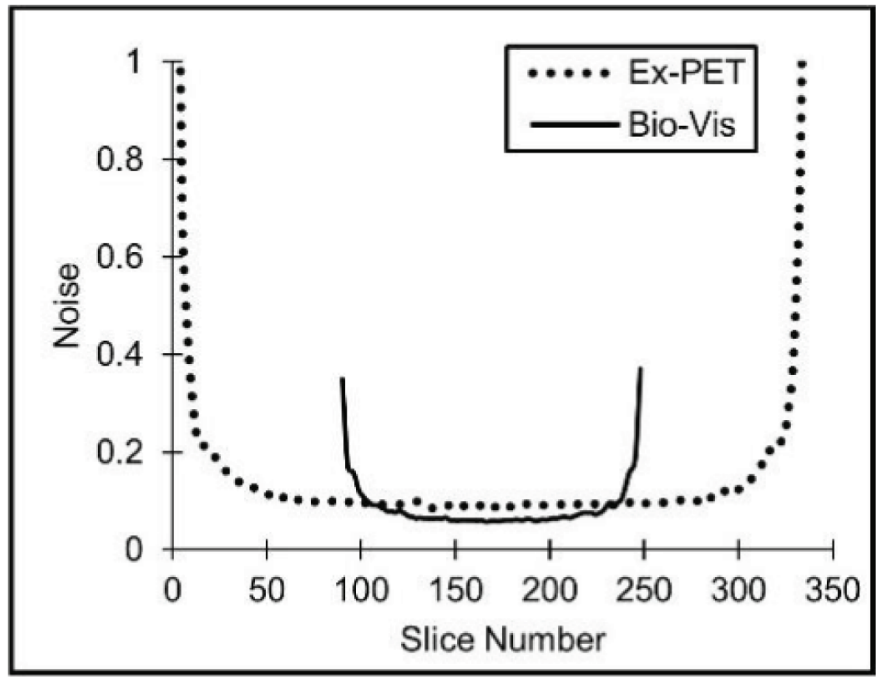

Fig. 9. Noise level per slice in a uniform cylinder phantom in the Bio-Vis and Ex-PET cases. The image noise levels vary more smoothly with Ex-PET versus the Bio-Vis configuration in the central overlapping section of the AFOV of the two systems (slice numbers 90-248).

effect due to gamma photons entering the scintillating crystals through the axial gaps. This can be accounted for by modeling the point spread function (PSF) response, as suggested by Schmall et al. [41], or by shielding the detector modules with, for example, tungsten rings in between the detector rings, a relatively economic and feasible solution which, however, may degrade to a small extent the system sensitivity. Our preliminary MC study (data not shown here) showed about $90 \%$ absorption of 511-keV gammas in a 1-cm thick tungsten layer which is a size that can be fitted within the proposed $3.2-\mathrm{cm}$ long axial gaps. Only a minor effect in spatial resolution and system sensitivity was observed with the Ex-PET after the introduction of shielding within the axial gaps.

Ex-PET demonstrated comparable system sensitivity to that of the Bio-Vis, which is expected due to the same number of detector elements enclosed in both systems. However, sparse detector rings configuration resulted in significant loss of counts in the AFOV regions corresponding to the gaps, and consequently in a reduction in axial sensitivity in these regions [Fig. 3(b)]. This axial imbalance in sensitivity between the gaps and the neighbouring physical rings was corrected with CBM acquisition [Fig. 3(c)], a standard technique on commercial Siemens Biograph PET/CT scanners that can be particularly useful for sparse detector module ring configurations of short or moderate AFOV lengths. The axial extent of CBM was set equal to twice the axial detector block width $(6.4 \mathrm{~cm})$ to allow uniform sampling of both axial edges of each gap region. CBM with a fixed bed speed was preferred 
TABLE VI

SF AND NECR PEAKS

\begin{tabular}{cccc}
\hline \hline & Bio-Vis & Ex-PET & Siemens Biograph Vision $^{\mathrm{TM}}$ \\
\hline SF & $34 \%$ & $34 \%$ & $37 \%$ \\
NECR Peak & $311 \mathrm{kcps}$ at $31.2 \mathrm{kBq} / \mathrm{mL}$ & $292 \mathrm{kcps}$ at $27.8 \mathrm{kBq} / \mathrm{mL}$ & $306 \mathrm{kcps}$ at $32 \mathrm{kBq} / \mathrm{mL}$ \\
\hline \hline
\end{tabular}

to multibed stationary acquisitions as it allowed the acquisition of a well-balanced amount of 3-D data between the gaps and the physical rings with the minimum involvement by the scanner operator and minimum number of bed starts and stops to minimize any discomfort in future patient studies. Besides, it is worth noting that CBM has also extended the Ex-PET active AFOV by $6.08 \mathrm{~cm}$, equivalent to the axial pitch of 19 detector elements or nearly two detector modules. Compared to the conventional compact ring configuration, the sparse detector rings configuration resulted in reduced sensitivity per unit axial length (volume sensitivity), and consequently in increased BV (maximum difference of $8 \%$ was observed for the 22-mm region: $3.18+/-0.07$ for Bio-Vis versus $3.44+/-0.58$ for Ex-PET; Table V). However, the percent CR was not affected, with less than $6 \%$ difference measured between the two scanner models (Table IV). The percent difference in CNR between the two system configurations improved as a function of scan time, decreasing from $18 \%$ at $1 \mathrm{~min}$ to $8 \%$ at $9 \mathrm{~min}$ for the $10-\mathrm{mm}$ sphere, and from $33 \%$ to $15 \%$ for the $37-\mathrm{mm}$ sphere (Fig. 7).

The proposed sparse geometry has been designed such that it offers more than $100 \%$ extension of the AFOV of conventional compact ring configuration with the same number of detector modules. As a result, it can achieve truly simultaneous static or dynamic PET imaging of distant organs, such as the brain and the heart or the gut and the heart, thus paving the way for novel imaging studies not previously possible with limited AFOV PET systems, including the investigation of the effect of specific molecular (e.g., inflammation, hypoxia, calcification, cell proliferation, etc.) or macroscopic mechanisms (e.g., blood flow or tracer perfusion) between distant organs or the image-based derivation of the blood plasma input function from a region distant from the targeted tissues. On the other hand, the proposed sparse configuration could allow the development of cost-effective low-cost clinical PET scanners with conventional the same AFOV lengths as that of modern clinical PET systems $(20-30 \mathrm{~cm})$ but with only $50 \%$ of the original volume of detector materials and thus at nearly half the cost. This will allow developing cost-effective PET scanners with conventional AFOV, and hence facilitate the worldwide adoption of this molecular imaging modality, particularly in regions with limited resources. Despite the expected reduction of system sensitivity by $\sim 75 \%$ in that case, the IQ performance may still not be significantly affected thanks to the application of limited-CBM 3-D PET sparse acquisitions [42], [43]. Although the sensitivity per slice is reduced by $\sim 50 \%$, the counts are spread out over longer AFOV.

However, in order to achieve this unique and highly important utilities, the system and volume sensitivity gains achieved by the more expensive long AFOV PET systems may no longer be attained, as discussed earlier. Consequently, although Ex-PET would be capable of both static and dynamic PET acquisitions across distant organs, the signal-to-noise ratio of the acquired data may be significantly lower thus preventing the ultrafast scan times or ultralow dosages achieved by the respectively, long AFOV PET systems with compact ring configurations. It is worthy to note that the sparse detector module configuration proposed in this study will increase the AFOV when fully extended to cover a large portion of the patient's body, yet it can be retracted to fully compact for high sensitivity single organ imaging making it more adaptable to variety of applications.

Nevertheless, the fact that Ex-PET attained the same system sensitivity over a longer AFOV than Bio-Vis also resulted in a smoother axial variance of the percentage $\mathrm{BV}$ at the overlapping section of the two systems AFOV (Fig. 9).

One limitation of this study is the lack of TOF, which was not included due to unavailability in the latest released version of STIR software. The implementation of the TOF timing resolution of 214 ps currently available on the Siemens Biograph Vision PET scanner [6] will enhance the signal-to-noise ratio and thus is expected to improve lesion detectability, compared to the non-TOF setting, thereby mitigating the differences in CNR between the sparse and compact ring datasets.

\section{CONCLUSION}

Sparse detector module rings configuration with CBM allows extending the current limited AFOV of conventional PET systems by more than $100 \%$ at no additional detector material costs and without significantly affecting NEMA CR, system sensitivity and transaxial spatial resolution. Although the system sensitivity remains intact, it is now covering nearly twice the original AFOV thus significantly reducing the volume sensitivity at the AFOV center. Finally, the axial spatial resolution is deteriorated as expected due to the increase in the axial parallax effect, as previously reported for other long AFOV PET systems.

\section{REFERENCES}

[1] D. L. Bailey, D. W. Townsend, P. E. Valk, and M. N. Maisey, Positron Emission Tomography. London, U.K.: Springer-Verlag, 2005.

[2] V. Bettinardi et al., "Performance evaluation of the new whole-body PET/CT scanner: Discovery ST," Eur. J. Nucl. Med. Mol. Imag., vol. 31, no. 6, pp. 867-881, 2004.

[3] J. A. Kolthammer, K. H. Su, A. Grover, M. Narayanan, D. W. Jordan, and R. F. Muzic, "Performance evaluation of the Ingenuity TF PET/CT scanner with a focus on high count-rate conditions," Phys. Med. Biol., vol. 59, no. 14, pp. 3843-3859, Jul. 2014.

[4] B. W. Jakoby, Y. Bercier, M. Conti, M. E. Casey, B. Bendriem, and D. W. Townsend, "Physical and clinical performance of the mCT time-of-flight PET/CT scanner," Phys. Med. Biol., vol. 56, no. 8, pp. 2375-2389, Apr. 2011.

[5] A. M. Grant, T. W. Deller, M. M. Khalighi, S. H. Maramraju, G. Delso, and C. S. Levin, "NEMA NU 2-2012 performance studies for the SiPMbased ToF-PET component of the GE SIGNA PET/MR system," J. Med. Phys., vol. 43, no. 5, p. 2334, May 2016. 
[6] J. van Sluis et al., "Performance characteristics of the digital biograph Vision PET/CT system," J. Nucl. Med., vol. 60, no. 7, pp. 1031-1036, Jan. 2019.

[7] D. F. C. Hsu, E. Ilan, W. T. Peterson, J. Uribe, M. Lubberink, and C. S. Levin, "Studies of a next-generation silicon-photomultiplierbased time-of-flight PET/CT system," J. Nucl. Med., vol. 58, no. 9, pp. 1511-1518, Sep, 2017.

[8] J. T. Thackeray, "Imaging the molecular footprints of the heart-brain axis in cardiovascular disease," J. Nucl. Med., vol. 60, no. 6, pp. 728-729, Jun. 2019.

[9] E. A. Mayer et al., "Role of brain imaging in disorders of braingut interaction: A rome working team report," Gut, vol. 68, no. 9, pp. 1701-1715, Sep. 2019.

[10] N. A. Karakatsanis, M. A. Lodge, A. K. Tahari, Y. Zhou, R. L. Wahl, and A. Rahmim, "Dynamic whole-body PET parametric imaging: I. Concept, acquisition protocol optimization and clinical application," Phys. Med. Biol., vol. 58, no. 20, pp. 7391-7418, Oct. 2013.

[11] S. R. Cherry, T. Jones, J. S. Karp, J. Qi, W. W. Moses, and R. D. Badawi, "Total-body PET: Maximizing sensitivity to create new opportunities for clinical research and patient care," J. Nucl. Med., vol. 59, no. 1, pp. 3-12, Jan, 2018

[12] S. Surti, A. R. Pantel, and J. S. Karp, "Total body PET: Why, how, what for?" IEEE Trans. Radiat. Plasma Med. Sci., vol. 4, no. 3, pp. 283-292, May 2020.

[13] R. D. Badawi et al., "First human imaging studies with the EXPLORER total-body PET scanner," J. Nucl. Med., vol. 60, no. 3, pp. 299-303, Mar. 2019.

[14] S. R. Cherry and J. Czernin, "Discussions with leaders: A conversation between Simon Cherry and Johannes Czernin," J. Nucl. Med., vol. 60, no. 3, pp. 295-298, Mar. 2019.

[15] V. Viswanath et al., "Development of PET for total-body imaging," Acta Phys Pol B, vol. 48, no. 10, pp. 1555-1566, 2017.

[16] J. S. Karp et al., "PennPET explorer: Design and preliminary performance of a whole-body imager," J. Nucl. Med., vol. 61, no. 1, pp. 136-143, Jan, 2020.

[17] M. E. Daube-Witherspoon, V. Vishwanath, M. E. Werner, and J. S. Karp, "Perfornance characteristics of long axial field-of-view PET scanners with axial gaps," IEEE Trans. Radiat. Plasma Med. Sci., early access, Sep. 28, 2020, doi: 10.1109/TRPMS.2020.3027257.

[18] N. Karakatsanis, S. Zein, and S. Nehmeh, "Performance evaluation of a clinical PET system with uniform axial gaps between individual detector rings," J. Nucl. Med., vol. 60, no. s1, p. 189, May 2019.

[19] T. Yamaya et al., "A proposal of an open PET geometry," Phys. Med. Biol., vol. 53, no. 3, pp. 757-773, Feb. 2008.

[20] N. A. Karakatsanis, S. A. Zein, and S. A. Nehmeh, "Evaluation of image quality and quantitation in a clinical PET scanner with a uniformly sparse detector rings configuration," in Proc. IEEE Nucl. Sci. Symp. Med. Imag. Conf., 2018, pp. 1-9.

[21] T. Yamaya et al., "Simulation studies of a new OpenPET geometry based on a quad unit of detector rings," Phys. Med. Biol., vol. 54, no. 5, pp. 1223-1233, 2009.

[22] T. Yamaya et al., "A multiplex 'OpenPET' geometry to extend axial FOV without increasing the number of detectors," IEEE Trans. Nucl. Sci., vol. 56, no. 5, pp. 2644-2650, Oct. 2009.

[23] E. Yoshida et al., "Development of a whole-body dual ring OpenPET for in-beam PET," IEEE Trans. Radiat. Plasma Med. Sci., vol. 1, no. 4, pp. 293-300, Jul. 2017.

[24] H. Tashima et al., "Development of a small single-ring OpenPET prototype with a novel transformable architecture," Phys. Med. Biol., vol. 61, no. 4, pp. 1795-1809, Feb. 2016.
[25] A. Salomon, D. Truhn, R. Botnar, F. Kiessling, and V. Schulz, "Sparse crystal setting and large axial FOV for integrated whole-body PET/MR," in Proc. IEEE Nucl. Sci. Symp. Conf., Oct. 2011, pp. 2521-2523.

[26] Z. Zhang et al., "Investigation of optimization-based reconstruction with an image-total-variation constraint in PET," Phys. Med. Biol., vol. 61, no. 16, pp. 6055-6084, Aug. 2016.

[27] J. Zhang, M. I. Knopp, and M. V. Knopp, "Sparse detector configuration in SiPM digital photon counting PET: a feasibility study," Mol. Imag. Biol., vol. 21, pp. 447-453, Aug. 2019.

[28] S. A. Zein, N. A. Karakatsanis, M. Issa, A. A. Haj-Ali, and S. A. Nehmeh, "Physical performance of a long axial field-of-view PET scanner prototype with sparse rings configuration: A Monte Carlo simulation study," Med. Phys., vol. 47, no. 4, pp. 1949-1957, 2020.

[29] V. Y. Panin, A. M. Smith, J. Hu, F. Kehren, and M. E. Casey, "Continuous bed motion on clinical scanner: design, data correction, and reconstruction," Phys. Med. Biol., vol. 59, no. 20, pp. 6153-6174, Sep. 2014.

[30] S. Jan et al., "GATE: A simulation toolkit for PET and SPECT," Phys. Med. Biol., vol. 49, no. 19, p. 4543, 2004.

[31] J. Allison et al., "Recent developments in GEANT4," Nucl. Instrum. Methods Phys. Res. A Accelerators Spectr. Detectors Assoc. Equip., vol. 835, pp. 186-225, Nov. 2016.

[32] R. Brun and F. Rademakers, "ROOT_An object oriented data analysis framework," Nucl. Instrum. Methods Phys. Res., vol. 389, nos. 1-2, pp. 81-86, 1997.

[33] M. Casey, H. Gadagkar, and D. Newport, "A component based method for normalization in volume PET," in Proc. Int. Meeting Fully 2-D Image Reconstruct. Radiol. Nucl. Med., vol. 1, 1996, pp. 67-71.

[34] NU 2-2012: Performance Measurements of Positron Emission Tomographs, Nat. Elect. Manuf. Assoc., Arlington, VA, USA, 2012.

[35] K. Thielemans et al., "STIR: Software for tomographic image reconstruction release 2," Phys. Med. Biol., vol. 57, no. 4, p. 867, 2012.

[36] M. E. Daube-Witherspoon, and G. Muehllehner, "Treatment of axial data in three-dimensional PET," J. Nucl. Med., vol. 28, no. 11, pp. 1717-1724, Nov. 1987.

[37] C. Lois et al., "An assessment of the impact of incorporating time-offlight information into clinical PET/CT imaging," J. Nucl. Med., vol. 51, pp. 237-245, Feb. 2010.

[38] G. S. Meirelles et al., "Deep-inspiration breath-hold PET/CT: Clinical findings with a new technique for detection and characterization of thoracic lesions," J. Nucl. Med., vol. 48, no. 5, pp. 712-719, May 2007.

[39] S. R. Cherry, R. D. Badawi, J. S. Karp, W. W. Moses, P. Price, and T. Jones, "Total-body imaging: Transforming the role of positron emission tomography," Sci. Transl. Med., vol. 9, p. 381, Mar. 2017.

[40] J. K. Poon et al., "Optimal whole-body PET scanner configurations for different volumes of LSO scintillator: A simulation study," Phys. Med. Biol., vol. 57, no. 13, pp. 4077-4094, Jul. 2012.

[41] J. P. Schmall, J. S. Karp, M. Werner, and S. Surti, "Parallax error in long-axial field-of-view PET scanners-a simulation study," Phys. Med. Biol., vol. 61, no. 14, pp. 5443-5455, Jul. 2016.

[42] N. A. Karakatsanis, S. A. Zein, and S. A. Nehmeh, "Positron emission tomography with sparse block rings and continuous bed motion," in Proc. IEEE Nucl. Sci. Symp. Med. Imag. Conf., Oct./Nov. 2019, pp. 1-6.

[43] N. A. Karakatsanis, S. A. Zein, and S. A. Nehmeh, "Continuous bed motion acquisition for clinical PET systems with a sparse block rings configuration," Eur. J. Nucl. Med. Mol. Imag., vol. 46, pp. S273-S274, Oct. 2019. 